\section{Câncer de colo de Útero: situação dos laboratórios em Alagoas}

Cervical cancer: state of laboratories to conduct tests in Alagoas Cáncer de cuello uterino: situación de los laboratorios en Alagoas

Heloisa Helena Motta Bandini ${ }^{1}$, Orcid Id no 0000-0002-7320-2637 Carmen Silvia Motta Bandini ${ }^{1}$ Orcid Id nº 0000-0002-4731-5785

Rodrigo Japur Duarte Tavares² Orcid Id no 0000-0001-8511-7520 Rozangela M. Almeida Fernandes Wyszomirska³ Orcid Id nํ0000-0003-0066-8927

1 Universidade Estadual de Ciências da Saúde de Alagoas, Maceió, AL, Brasil.

${ }^{2}$ Centro Universitário Serra dos Órgãos, Teresópolis, RJ Brasil.

3Universidade Federal de Alagoas, Maceió, AL, Brasil.

\section{Resumo}

O câncer de colo de útero tem alta incidência entre as mulheres brasileiras e o exame citopatológico de colo de útero (Teste de Papanicolaou) é uma importante ferramenta para o seu combate por identificar as lesões precursoras da doença. Avaliar a qualidade interna dos laboratórios que realizam os exames citopatológicos de colo de útero para o SUS em Alagoas de modo a permitir o monitoramento dos serviços prestados. Para tanto, foram utilizados os indicadores de controle interno de qualidade dos laboratórios (índice de positividade, percentual de exames compatíveis com ASC entre os exames satisfatórios, percentual de ASC entre os exames alterados, razão ASC/SIL, percentual de exames compatíveis com HSIL e percentual de amostras insatisfatórias), para o triênio 2011-2013, com base nos dados disponíveis no SISCOLO. A qualidade dos laboratórios investigados está abaixo da recomendada pelas organizações de saúde e Alagoas apresenta uma cobertura de exames inferior à necessária para a população. Os resultados sugerem falhas do sistema de saúde e a necessidade de replanejamento de todo o ciclo de prevenção e controle do câncer de colo de útero no Estado.

Descritores: Teste de Papanicolaou, Neoplasias de colo do útero, Câncer.

\section{Abstract}

Cervical cancer has a high incidence among Brazilian women and the cytopathological exam of the uterine cervix (Pap test) is an important tool in its combat by identifying the precursor lesions of the disease. OBJECTIVE: evaluate the internal quality of the laboratories that perform cervical cytopathology tests for the UHS in Alagoas in order to allow the monitoring of the services provided. METHOD: To do so, we used the internal quality control indicators of the laboratories (positivity index, percentage of tests compatible with ASC among satisfactory tests, percentage of ASC among altered tests, ASC/SIL ratio, percentage of tests compatible with HSIL and percentage of unsatisfactory samples), for the triennium 2011-2013, based on data available in SISCOLO. RESULT: the quality of the laboratories investigated is below that recommended by the health organizations and Alagoas has a lower coverage of exams than the population needs. CONCLUSION: the results suggest failures in the health system and the need for redesigning the entire cycle of cervical cancer prevention and control in the state.

Descriptors: Papanicolaou Test; Uterine Cervical Neoplasms; Cancer.

\section{Resumen}

El cáncer de cuello uterino tiene una alta incidencia entre las mujeres brasileñas y el examen citopatológico del cuello de útero (Prueba de Papanicolaou) es una herramienta importante para su combate, ya que identifica lesiones precursoras de la enfermedad. Evaluar la calidad interna de los laboratorios que realizan exámenes citopatológicos de cuello de útero para el SUS en Alagoas a fin de permitir el monitoreo de los servicios prestados. Para ello se utilizaron indicadores de control interno de calidad de los laboratorios (índice de positividad, porcentaje de pruebas compatibles con ASC entre pruebas satisfactorias, porcentaje de ASC entre pruebas alteradas, relación ASC / SIL, porcentaje de pruebas compatibles con HSIL y porcentaje de muestras insatisfactorias) para el período 2011-2013, con base en los datos disponibles en SISCOLO. La calidad de los laboratorios investigados está por debajo de la recomendada por las organizaciones de salud y Alagoas tiene una cobertura de exámenes por inferior a la necesaria para la población. Los resultados sugieren fallas del sistema de salud y la necesidad de rediseñar todo el ciclo de prevención y control del cáncer de cuello de útero en el Estado.

Descriptores: Prueba de Papanicolaou, Neoplasias de Cuello de Útero, Cáncer. 


\section{Introdução}

O câncer de colo de útero é o quarto tipo de câncer mais incidente entre as mulheres em todo o mundo ${ }^{1}$. A taxa de mortalidade por esse tipo de câncer, no país, é muito superior à de países desenvolvidos ${ }^{2}$, indicando uma provável falha na detecção precoce.

Desde 1941, o exame citopatológico tem sido considerado como uma importante ferramenta de detecção do câncer de colo de útero por identificar as lesões precursoras do câncer ainda em sua fase tratável $^{3}$. Contudo, o exame apresenta algumas limitações, tais como a baixa sensibilidade e a grande quantidade de amostras insatisfatórias, além de resultados falsos negativos ocasionados por problemas na coleta do material, na fixação e no preparo dos esfregaços e até mesmo no treinamento do examinador ${ }^{4}$ 5. Os erros cometidos na coleta são responsáveis por $20 \%$ a $39 \%$ dos resultados falsos negativos devido a erros de escrutínio ${ }^{3}$, quando as células neoplásicas estão representadas no esfregaço, porém, não são reconhecidas pelo escrutinador; ou a não representatividade ou a escassez de células neoplásicas na amostra e a presença de fundo necrótico ou inflamação nos esfregaços, que prejudicam a análise ${ }^{6}$.

Para garantir a qualidade dos exames, faz-se necessário tanto um controle interno como externo ${ }^{7}$. O controle externo é realizado por um segundo laboratório que reanalisa, de forma cega, 10\% das lâminas normais e todas as lâminas alteradas do laboratório inicial. Este parâmetro é utilizado no Brasil desde o final de $2013^{8}$. Já o controle interno deve ser realizado pelo próprio laboratório e é definido como um conjunto de ações sistematizadas e realizadas regularmente com a finalidade de monitorar a adequabilidade da amostra, 0 tempo de escrutínio, o controle da carga de trabalho do escrutinador, a revisão hierárquica dos esfregaços e a revisão dos esfregaços negativos ${ }^{9}$.

Cabe, aos gestores estadual e municipal, a fiscalização da qualidade dos serviços prestados à população usuária do Sistema Único de Saúde (SUS), seja esse prestador de serviço público ou privado7. O Sistema de Informação do Câncer do Colo de Útero (SISCOLO) foi um valioso instrumento de gestão para os
Estados e municípios no monitoramento da qualidade dos serviços dos laboratórios de citologia, sendo descontinuado e implantado o SISCAN. No entanto, o novo sistema passa por dificuldades operacionais, sendo os dados de 2013 do SISCOLO os últimos que podem dar indícios da qualidade dos serviços de citologia oferecidos à população usuária do SUS até o momento.

Alguns estudos vêm sendo conduzidos com o objetivo de verificar a qualidade dos exames citopatológicos realizados na rede pública no país. Em 2012, por exemplo, Bortolon et al. ${ }^{10}$ empreenderam uma pesquisa nacional avaliando quatro indicadores de qualidade interna dos laboratórios que realizavam 0 referido exame (índice de positividade, percentual de exames compatíveis com lesão intraepitelial de alto grau, percentual de exames compatíveis com atipias de significado indeterminado em células escamosas e razão de atipias escamosas de significado indeterminado por lesões intraepiteliais escamosas). O estudo utilizou os dados do SISCOLO referentes ao período entre janeiro e dezembro de 2010 e verificou um alto percentual de laboratórios com índices de positividade considerados muito baixos, principalmente nas regiões Norte, Sul e Nordeste do país (54\%, 57\% e 59\%, respectivamente). Para os autores, esses dados podem indicar a não identificação de amostras positivas, gerando, assim, a emissão de muitos laudos com falsos negativos.

Apesar da importância do mapeamento da efetividade dos laboratórios que prestam serviços ao SUS com a realização dos exames citopatológicos, verifica-se que não existem publicações recentes sobre o assunto no Brasil. Em que pese a discussão recente sobre a avaliação e o planejamento no âmbito da Saúde Coletiva ${ }^{11-13}$ é preciso que a avaliação dos laboratórios, ocorrendo de forma anterior ou em conjunto com o planejamento do serviço, possa ser uma fonte de orientação da gestão pública na tentativa de sanar os constantes problemas do atendimento à população. $\mathrm{O}$ Estado de Alagoas não possui pesquisa abrangendo a rede como um todo e que avalie os indicadores de qualidade interna, evidenciando, assim, uma lacuna na literatura e, principalmente, uma dificuldade extra para a 
gestão de saúde estadual.

Diante do exposto, o objetivo deste estudo foi avaliar a qualidade interna dos laboratórios de citologia que prestam serviços para o SUS para o Estado de Alagoas de modo a permitir o monitoramento dos serviços prestados, bem como auxiliar no planejamento das ações estruturantes de combate ao câncer de colo de útero no Estado.

\section{Método}

Fizeram parte deste estudo os dados coletados no SISCOLO para os anos de 2011, 2012 e 2013 de laboratórios credenciados para a realização dos exames citopatológicos pelo SUS no Estado de Alagoas. Como forma de evitar distorções na amostra, os dados foram avaliados ano a ano e em conjunto.

Dos dados disponíveis no SISCOLO, foram analisados cinco indicadores do Controle Interno detalhados abaixo.

\section{Índice de Positividade (IP)}

O IP ( $n^{\circ}$ de exames alterados em determinado local e ano/total de exames satisfatórios $x$ 100) tem como finalidade determinar a prevalência de alterações celulares nos exames citológicos e indicar a sensibilidade do exame no rastreamento para detectar as lesões na população avaliada. Considera-se muito baixa a categorização do IP inferior a 2,0\%; baixa, a localizada entre $2,0 \%$ e 2,9\%; esperada, a localizada entre $3,0 \%$ e $10 \%$ e acima do esperado, a superior a $10 \%{ }^{14}$.

\section{Percentual de exames compatíveis com ASC entre os exames satisfatórios (ASC/Sat)}

ASC-US e ASC-H são siglas para identificar as células escamosas atípicas de significado indeterminado. Este indicador representa que os achados citológicos não são suficientes para 0 diagnóstico de lesão intraepitelial. Foi calculado por meio da fórmula $n^{\circ}$ de exames com ASC-US e ASC-H/total de exames satisfatórios $x$ 100. A recomendação é que o percentual de ASC não seja superior a $5 \%$ dos exames satisfatórios $^{14}$.
Percentual de ASC entre os exames alterados (ASC/Alt)

O objetivo deste indicador é avaliar o percentual de células escamosas atípicas de significado indeterminado (ASC) entre os exames alterados. Em conjunto com o IP, este indicador pode demonstrar ambiguidade citológica, uma vez que um valor adequado de IP pode conter um elevado percentual de exames compatíveis com ASC. Foi calculado por meio da fórmula $n^{\circ}$ de exames com ASC-US e ASC-H/total de exames alterados $x$ 100. O percentual de células escamosas atípicas de significado indeterminado entre os exames alterados deve ser inferior a $60 \%{ }^{15}$.

\section{Razão ASC/SIL (ASC/SIL)}

A razão $\mathrm{ASC} / \mathrm{SIL}$ ( $n^{\circ}$ de ex. compatíveis com ASC-US e ASC-H/ $n^{\circ}$ de exames com LSIL e HSIL $x$ 100) avalia a qualidade técnica do laboratório em verificar o diagnóstico diferencial de lesões intraepiteliais escamosas (LSIL e HSIL). As recomendações são para manter uma relação não superior a 3:1 $1^{14}$.

\section{Percentual de exames compatíveis com HSIL}

Esse indicador, $n^{\circ}$ de exames HSIL/total de exames satisfatórios $x$ 100, mede a capacidade de detecção de lesões precursoras do câncer e deve ter um percentual igual ou superior a $0,4 \%$ dos exames satisfatórios ${ }^{15}$. Este é o principal indicador deste conjunto de indicadores, pois as lesões intraepiteliais de alto grau são aquelas que têm um grande potencial de progressão para o câncer do colo do útero.

Todos os dados utilizados eram públicos e estavam disponíveis na base de dados do DATASUS/SISCOLO para o período e processados pelo software Microsoft Excel, versão 2016.

A nomenclatura dos esfregaços adotada no estudo foi a utilizada no Sistema de Bethesda ${ }^{16}$. Neste caso, ASC corresponde à nomenclatura para células escamosas atípicas; ASC - US, para as células escamosas atípicas de significado indeterminado; ASC$\mathrm{H}$ significa a presença de células escamosas atípicas, que não permitem excluir uma lesão de alto grau; SIL indica uma lesão intraepitelial escamosa; HSIL significa lesão intraepitelial escamosa de alto grau e, por fim, 
LSIL corresponde à nomenclatura para lesão intraepitelial escamosa de baixo grau. As fórmulas utilizadas para o cálculo dos indicadores e as descrições de cada indicador foram as mesmas utilizadas por Plewka et al. ${ }^{17}$.

\section{Resultados}

No triênio estudado, os laboratórios avaliados realizaram 276.738 exames, totalizando $86,4 \%$ dos exames realizados no Estado. Quando se observou a quantidade de exames realizados por cada laboratório, verificou-se que, em 2011, apenas dois laboratórios $(8,6 \%)$ atingiram o parâmetro recomendado pelo
Ministério da Saúde, o qual estabelece 15 mil exames/ano no mínimo. Nos dois anos seguintes, somente um laboratório (4,1\% para 2012 e 3,5\% para 2013) atingiu o parâmetro em questão.

As tabelas 1, 2 e 3 apresentam os resultados da análise das variáveis estudadas. O IP demonstrou que, no ano de 2011, aproximadamente $20 \%$ dos laboratórios apresentavam índices dentro do esperado. Para o ano de 2012, os resultados foram discretamente mais altos, com 25\% dos laboratórios com IP considerados dentro ou acima do esperado. O ano de 2013 apresentou os melhores resultados para o triênio: cerca de $30 \%$ dos serviços apresentaram IP dentro do esperado.

Tabela 1- Indicadores de qualidade interna dos laboratórios em Alagoas no ano de 2011.

\begin{tabular}{|c|c|c|c|c|c|c|c|}
\hline Laboratório & Quantidade de Exames & $\overline{\text { IP }}$ & ASC/Sat & ASC/ Alt & ASC/SIL & "HSIL & Insat \\
\hline 1 & 18.468 & 1,61 & $0,78 \%$ & $48,38 \%$ & 1,16 & $0,23 \%$ & 6,78 \\
\hline 2 & 3.443 & 1,92 & $1,07 \%$ & $56,06 \%$ & 1,42 & $0,26 \%$ & 0,03 \\
\hline 3 & 3.122 & 0,16 & $0,00 \%$ & $0,00 \%$ & 0,00 & $0,00 \%$ & 0,03 \\
\hline 4 & 9.115 & 1,56 & $0,42 \%$ & $27,14 \%$ & 0,41 & $0,12 \%$ & 1,70 \\
\hline 5 & 465 & 0,86 & $0,22 \%$ & $25,00 \%$ & 0,50 & $0,43 \%$ & 0,00 \\
\hline 6 & 1.444 & 5,39 & $3,69 \%$ & $68,42 \%$ & 2,89 & $0,50 \%$ & 2,42 \\
\hline 7 & 47.438 & 0,54 & $0,33 \%$ & $60,71 \%$ & 1,89 & $0,03 \%$ & 1,32 \\
\hline 8 & 2.425 & 1,66 & $0,95 \%$ & $57,50 \%$ & 1,35 & $0,17 \%$ & 0,54 \\
\hline 9 & 1.004 & 1,49 & $1,39 \%$ & $93,33 \%$ & 14,00 & $0,00 \%$ & 0,00 \\
\hline 10 & 2.754 & 0,91 & $0,44 \%$ & $48,00 \%$ & 2,00 & $0,22 \%$ & 0,04 \\
\hline 11 & 12.616 & 0,03 & $0,01 \%$ & $25,00 \%$ & 0,00 & $0,00 \%$ & 0,00 \\
\hline 12 & 1.579 & 2,47 & $1,90 \%$ & $76,92 \%$ & 3,75 & $0,25 \%$ & 0,00 \\
\hline 13 & 126 & 4,81 & $0,96 \%$ & $20,00 \%$ & 0,25 & $0,00 \%$ & 17,46 \\
\hline 14 & 675 & 1,63 & $0,74 \%$ & $45,45 \%$ & 1,00 & $0,44 \%$ & 0,00 \\
\hline 15 & 3.530 & 1,79 & $0,99 \%$ & $55,56 \%$ & 1,30 & $0,23 \%$ & 0,14 \\
\hline 16 & 2.230 & 0,18 & $0,00 \%$ & $0,00 \%$ & 0,00 & $0,00 \%$ & 0,13 \\
\hline 17 & 199 & 7,07 & $2,53 \%$ & $35,71 \%$ & 0,56 & $0,51 \%$ & 0,50 \\
\hline 18 & 406 & 6,65 & $6,16 \%$ & $92,59 \%$ & 5,00 & $0,74 \%$ & 0,00 \\
\hline 19 & 1.477 & 1,83 & $0,81 \%$ & $44,44 \%$ & 0,86 & $0,27 \%$ & 0,07 \\
\hline 20 & 2.007 & 1,42 & $1,16 \%$ & $82,14 \%$ & 4,60 & $0,05 \%$ & 1,49 \\
\hline 21 & 119 & 2,52 & $1,68 \%$ & $1,60 \%$ & 2,00 & $0,00 \%$ & 0,00 \\
\hline
\end{tabular}

Fonte: SISCOLO - DATASUS.

Em relação ao ASC/Sat, para os anos de 2011e 2012, a quase totalidade dos serviços, exceto os executados pelo laboratório 18, apresentou ASC/Sat dentro do esperado. Em relação ao ASC/Alt, os resultados para 2012 e 2013 mostram que apenas 50\% dos serviços estavam dentro dos padrões para ASC/Alt.

O quarto indicador, ASC/SIL, demonstrou que, no ano de 2011, cerca de $81 \%$ dos serviços apresentaram um resultado dentro do esperado, enquanto, nos anos de 2012 e 2013, os resultados foram considerados dentro do esperado em $75 \%$ e $87,5 \%$ dos laboratórios, respectivamente. No entanto, os resultados obtidos pelo laboratório 9 para os anos de 2011 e 2012 (Tabelas 1 e 2) chamam a atenção pelo alto número de
ASC para cada caso de SIL. Para 2011, o laboratório 9 apresentou 14 ASC para cada caso de SIL e, em 2012, foram nove casos de ASC para cada caso de SIL. O mesmo pode ser observado para o laboratório 22, no ano de 2013 (Tabela 3), com sete casos de ASC para cada caso de SIL.

O quinto indicador de qualidade interna calculado foi o HSIL, em que apenas o laboratório 6 atingiu níveis aceitáveis, de $0,44 \%$, em 2012. Para os anos de 2011 e 2013, cerca de $25 \%$ dos laboratórios atingiram níveis adequados de HSIL.

Os dados da tabela 4 apresentam uma análise do triênio 2011-2013 geral do desempenho de Alagoas 
para os quatro índices avaliados. O IP médio do Estado no período foi de 2,29\% e o HSIL, de 0,18\%.

Tabela 2 - Indicadores de qualidade interna dos laboratórios em Alagoas no ano de 2012.

\begin{tabular}{|c|c|c|c|c|c|c|c|}
\hline Laboratório & Quantidade de Exames & IP & ASC/Sat & ASC/ Alt & ASC/SIL & $\overline{\mathrm{HSIL}}$ & Insat \\
\hline 1 & 11.777 & 2,22 & 1,16 & 52,08 & 1,44 & 0,22 & 8,19 \\
\hline 2 & 3.121 & 2,05 & 1,19 & 57,81 & 1,95 & 0,26 & 0,00 \\
\hline 3 & 6.695 & 0,21 & 0,03 & 14,29 & 0,22 & 0,00 & 0,13 \\
\hline 4 & 8.431 & 1,90 & 0,63 & 33,33 & 0,52 & 0,14 & 0,82 \\
\hline 5 & 1.401 & 0,07 & 0,00 & 0,00 & 0,00 & 0,00 & 0,14 \\
\hline 6 & 1.828 & 5,46 & 3,48 & 63,64 & 2,17 & 0,44 & 0,88 \\
\hline 7 & 22.047 & 0,50 & 0,33 & 66,36 & 2,70 & 0,05 & 0,26 \\
\hline 8 & 2.573 & 3,03 & 2,26 & 74,36 & 3,22 & 0,08 & 0,04 \\
\hline 9 & 1.569 & 1,34 & 1,21 & 90,48 & 9,50 & 0,13 & 0,00 \\
\hline 10 & 2.044 & 0,78 & 0,54 & 68,75 & 2,20 & 0,15 & 0,05 \\
\hline 11 & 10.070 & 0,05 & 0,02 & 40,00 & 0,00 & 0,00 & 0,00 \\
\hline 12 & 1.220 & 1,97 & 1,56 & 79,17 & 3,80 & 0,16 & 0,00 \\
\hline 13 & 381 & 0,62 & 0,00 & 0,00 & 0,00 & 0,00 & 15,49 \\
\hline 14 & 858 & 1,05 & 0,35 & 33,33 & 0,60 & 0,23 & 0,47 \\
\hline 15 & 1.567 & 1,09 & 0,89 & 82,35 & 4,67 & 0,19 & 0,06 \\
\hline 17 & 1.005 & 3,58 & 2,19 & 61,11 & 1,69 & 0,10 & 0,00 \\
\hline 18 & 1.794 & 10,14 & 8,53 & 84,07 & 5,67 & 0,00 & 0,00 \\
\hline 19 & 1.547 & 1,68 & 0,97 & 57,69 & 2,50 & 0,06 & 0,19 \\
\hline 21 & 999 & 4,51 & 3,01 & 66,67 & 2,73 & 0,20 & 0,10 \\
\hline
\end{tabular}

Tabela 3 - Indicadores de qualidade interna dos laboratórios em Alagoas no ano de 2013.

\begin{tabular}{|c|c|c|c|c|c|c|c|}
\hline Laboratório & Quantidade de Exames & IP & ASC/Sat & ASC/Alt & ASC/SIL & HSIL & Insat \\
\hline 1 & 3081 & 1,44 & 0,49 & 34,21 & 0,59 & 0,49 & 14,15 \\
\hline 2 & 3642 & 3,57 & 1,92 & 53,85 & 1,52 & 0,58 & 0,05 \\
\hline 3 & 4235 & 0,80 & 0,28 & 35,29 & 0,55 & 0,07 & 0,05 \\
\hline 4 & 5688 & 2,47 & 0,88 & 35,71 & 0,60 & 0,16 & 0,49 \\
\hline 5 & 870 & 0,81 & 0,00 & 0,00 & 0,00 & 0,00 & 0,11 \\
\hline 6 & 1546 & 7,99 & 3,34 & 41,80 & 1,42 & 0,92 & 1,23 \\
\hline 7 & 29919 & 2,35 & 1,47 & 62,45 & 2,01 & 0,15 & 0,20 \\
\hline 8 & 2331 & 5,83 & 4,59 & 78,68 & 3,96 & 0,30 & 0,00 \\
\hline 9 & 881 & 1,14 & 1,14 & 100,00 & 0,00 & 0,00 & 0,00 \\
\hline 10 & 3536 & 1,24 & 0,88 & 70,45 & 2,82 & 0,11 & 0,00 \\
\hline 11 & 2795 & 0,04 & 0,04 & 100,00 & 0,00 & 0,00 & 0,29 \\
\hline 12 & 1435 & 2,09 & 1,39 & 66,67 & 2,50 & 0,21 & 0,00 \\
\hline 14 & 3134 & 8,39 & 4,09 & 48,67 & 1,03 & 0,54 & 0,03 \\
\hline 15 & 1337 & 5,56 & 3,38 & 60,81 & 1,67 & 0,83 & 0,52 \\
\hline 16 & 770 & 0,00 & 0,00 & 0,00 & 0,00 & 0,00 & 0,00 \\
\hline 17 & 1099 & 5,74 & 2,64 & 46,03 & 3,63 & 0,55 & 0,18 \\
\hline 18 & 1631 & 2,70 & 2,51 & 93,18 & 0,00 & 0,00 & 0,00 \\
\hline 19 & 1786 & 1,24 & 0,68 & 54,55 & 2,00 & 0,06 & 1,01 \\
\hline 21 & 1265 & 3,08 & 2,13 & 69,23 & 3,00 & 0,00 & 0,00 \\
\hline 22 & 6376 & 0,25 & 0,22 & 87,50 & 7,00 & 0,03 & 0,38 \\
\hline 23 & 614 & 0,33 & 0,33 & 100,00 & 0,00 & 0,00 & 0,00 \\
\hline
\end{tabular}

Fonte: SISCOLO - DATASUS.

Tabela 4 - Média dos indicadores de qualidade interna dos laboratórios em Alagoas por ano e para o triênio 2011 a 2013.

\begin{tabular}{ccccccc}
\hline Ano & IP & ASC/Sat & ASC/Alt & ASC/SIL & HSIL & Insat \\
\hline 2011 & 2,21 & $1,25 \%$ & $45,90 \%$ & 2,14 & $0,21 \%$ & $1,56 \%$ \\
2012 & 2,16 & 1,44 & $53,27 \%$ & 2,31 & $0,12 \%$ & $1,34 \%$ \\
2013 & 2,51 & 1,39 & $55,80 \%$ & 1,46 & $0,21 \%$ & $1,09 \%$ \\
Triênio & 2,29 & $1,36 \%$ & $51,66 \%$ & 1,97 & $0,18 \%$ & $1,33 \%$ \\
\hline \multicolumn{7}{c}{ Fonte: SISCOLO - DATASUS. }
\end{tabular}

\section{Discussão}

Foi observado que a quantidade de exames realizados anualmente por cada laboratório estava abaixo da preconizada pelo Ministério da Saúde, provavelmente devido a dois fatores. O primeiro estaria relacionado com a pulverização de exames entre os laboratórios que prestavam serviços para o SUS em Alagoas. No período de 2011 a 2013, em número de $22^{18}$, enquanto 17 laboratórios seriam suficientes para 
suprir a demanda do Estado de Alagoas ${ }^{7}$. O segundo fator a ser considerado é a baixa cobertura de exames de citologia na população alagoana (média de 14,25\%) para o período de 2011 a 2013. Dados do Ministério da Saúde apontam que a razão de exames citopatológicos em Alagoas para os anos de 2011, 2012 e 2013 foi de 0,49, 0,47 e 0,43, respectivamente ${ }^{19}$. Esses resultados estão abaixo do esperado e do pactuado em 2012, no Pacto pela Saúde ${ }^{15}$, cuja meta para o ano era uma razão de 0,75 de exames citopatológicos.

O primeiro indicador avaliado foi o IP. As análises dos dados avaliados indicam que, para o triênio estudado, a maior parte dos exames realizados em Alagoas foi empreendida por unidades laboratoriais cujo IP estava aquém do recomendado. Os resultados são compatíveis com os observados por Plewka et al. ${ }^{17} \mathrm{e}$ Bortolon et al. ${ }^{10} \mathrm{e}$ indicam que medidas precisam ser tomadas para que os laboratórios escolhidos para a realização dos exames citopatológicos de colo de útero no Estado tenham qualidade superior. Medidas, como o aumento da qualidade do pré-escrutínio de todos os esfregaços ou a inclusão de revisão rápida de 100\% dos esfregaços negativos, podem ser benéficas nesse sentido.

Ao observar os dados sobre o IP apresentados na tabela 1, é possível verificar um dado preocupante, qual seja, o de que os laboratórios 1, 7 e 11 realizaram, juntos, 68,4\% dos exames de 2011 e 55\% dos exames de 2012. Todos esses serviços apresentaram um IP considerado muito baixo. Em 2013 (Tabela 3), cerca de $38,6 \%$ dos exames foram realizados pelo laboratório 7 e este apresentou IP de 2,35, considerado baixo, porém, superior ao IP apresentado nos anos anteriores.

Os achados relacionados ao Percentual de Exames Compatíveis com ASC entre os Exames Satisfatórios (ASC/Sat), para o triênio 2010-2013em Alagoas, demonstram que boa parte dos exames apresentou células escamosas atípicas de significado indeterminado, ou seja, resultados para os quais não se pode confirmar ou descartar um diagnóstico de lesão intraepitelial. Se forem avaliados, em conjunto, a média de percentual de ASC/Alt para o triênio com os indicadores de IP e o Percentual de Exames Compatíveis com Lesão de Alto Grau (HSIL), pode-se verificar que $83,4 \%$ dos serviços avaliados em Alagoas, no triênio 2011-2013, não têm clareza na identificação dos critérios citomorfológicos das lesões intraepiteliais, tanto de baixo como de alto grau. Esses resultados são semelhantes aos de Plewka et al. ${ }^{17} \mathrm{e}$ mostram que os diagnósticos realizados pelos laboratórios de citopatologia em Alagoas não apresentam certeza dos resultados encontrados e não são capazes de identificar, com clareza, se os exames indicam ou não lesões precursoras do câncer de colo de útero. Dessa forma, é viável a inferência de que muitas das lâminas com resultados indeterminados tinham grande chance de ser positivas e, com isso, perdeu-se a oportunidade de se intervir com urgência nas lesões precursoras do câncer, deixando-se para detectá-lo somente quanto a doença se mostrasse em fases mais avançadas.

Ao observar os dados relacionados à mortalidade por câncer de colo de útero em Alagoas no mesmo período, pode-se verificar um aumento em relação ao período anterior. Dados do $\mathrm{INCA}^{20}$, para o período de 2005-2009, indicavam uma taxa de mortalidade de 9,78\%, enquanto, no período de 20102014, a taxa subiu para 10,95\%. Estes resultados estão abaixo dos resultados encontrados na região Nordeste para o período, no entanto, são muito superiores aos de países desenvolvidos $20-21$.

Uma questão deve ser levantada a respeito do laboratório 18. Os resultados de IP para este laboratório foram acima do esperado ${ }^{14}$. Normalmente, resultados como estes são obtidos em serviços especializados de referência, o que não é o caso do laboratório, tendo em vista que os laboratórios de referência para Alagoas (laboratórios tipo II) eram outros dois ${ }^{18}$. Nesse caso, não se descarta a possibilidade de que tenha havido algum erro de alimentação do SISCOLO por parte do laboratório, especialmente se for considerado que 0 Percentual de Exames Compatíveis com ASC entre os Exames Satisfatórios (ASC/Sat) para o laboratório 18 estava abaixo do adequado, indicando ambiguidade citológica clara na análise das lâminas.

Outros resultados que apontam falhas na avaliação das lâminas são a análise da Razão entre os Exames Indeterminados e os Confirmados com Lesão de Alto Grau (ASC/SIL) e o Percentual de Exames 
Compatíveis com Lesões de Alto Grau (HSIL). Os resultados indicam que, para cada caso de exame sem certeza diagnóstica, apenas um caso foi confirmado com lesão de alto grau, o que é abaixo do recomendado ${ }^{14}$. Para evitar os casos de dúvidas ou de falsos negativos de erros nas leituras dos exames, Tavares et al. ${ }^{3}$ sugeriram que uma revisão de $100 \%$ das lâminas negativas deve ser implantada, ainda que esta solução consuma mais recursos e tempo.

Por fim, uma última questão precisa ser tratada: como as avaliações constantes do sistema passarão a gerar mudanças reais na prestação de serviços à população e na própria lógica de atuação das equipes envolvidas? Como se vê nos resultados aqui apresentados, a constante aplicação dos indicadores de controle interno dos laboratórios e a alimentação dos sistemas informatizados de dados das Secretarias de Estado/Ministério da Saúde mostram que há uma institucionalização do processo de avaliação, todavia, as informações ali encontradas permanecem desconectadas da atuação nos próprios serviços avaliados. Apesar do aumento inegável dos sistemas de avaliação do poder público sobre os serviços em saúde, não parece haver melhoria nos serviços em questão, nem para os que atendem, nem para os que são atendidos ${ }^{11}$.

Além disso, mesmo com um considerável aumento na quantidade de estudos acerca do tema avaliação e planejamento de políticas públicas em saúde no Brasil, raras foram as vezes em que as discussões científicas na área serviram de guia para a tomada de decisões práticas que ultrapassassem um modelo voltado apenas para a eficiência do sistema, em que os resultados são friamente analisados em relação às metas iniciais do serviço ${ }^{12}$. O fato é que os autores entendem que a avaliação deve guiar a tomada de decisões, levando-se em conta o real compromisso com a capacitação permanente das equipes envolvidas, a autonomia dos grupos de atores do processo e a proposição de apoio institucional para os menos aptos, na tentativa de correção dos problemas verificados. No caso dos exames citopatológicos, essas considerações parecem ser de especial importância, dado que a formação constante e o apoio institucional poderiam diminuir os erros na coleta e de escrutínio das lâminas, bem como controlar adequadamente a quantidade de trabalho do escrutinador, minimizando muitos dos fatores considerados responsáveis por muitos dos falsos negativos encontrados 3 , .

\section{Conclusões}

Os resultados deste estudo demonstram que a qualidade interna dos laboratórios que realizaram exames citopatológicos para o SUS em Alagoas está abaixo da recomendada. Os dados também mostraram que Alagoas falha em dois quesitos essenciais no combate ao câncer: apresenta uma cobertura de exames citopatológicos cervicais abaixo da necessária para a população e oferece serviços de avaliação dos exames realizados com qualidade abaixo da recomendada pelas autoridades de saúde.

Nesse cenário, parece ser necessária uma intervenção urgente das autoridades estaduais no estabelecimento do controle interno e externo das práticas estabelecidas pelos laboratórios de citopatologia, contudo, não apenas em uma lógica reducionista relativa à adequação resultado-meta, mas em um modelo que possa colocar a constante avaliação dos indicadores como uma ferramenta na criação de estratégias de transformação do sistema de saúde.

\section{Referências}

1. Ministério da Saúde (BR), Instituto Nacional do Câncer José Alencar da Silva. Estimativa 2018: Incidência de Câncer no Brasil [Internet]. Rio de Janeiro: INCA; 2018 [cited 2020 Aug 10]. Available from: http://www1.inca.gov.br/rbc/n_64/v01/pdf/15resenha-estimativa-2018-incidencia-de-cancer-no-brasil.pdf

2. World Health Organization, International Agency for Research on Cancer. Globocan 2012: Estimated Cancer Incidence, Mortality and Prevalence Worldwide in 2012 v1.0 [Internet]. Geneva: WHO; 2021 [cited 2020 Aug 10]. Available from:

https://publications.iarc.fr/Databases/l

3. Tavares SBN, Amaral RG, Manrique EJC, Sousa NLA, Albuquerque ZBP, Zeferino LC. Quality control in cervica cytopathology: a literature review. Rev Bras Cancerol [Internet]. 2007 July/Sep [cited 2020 Aug 10]; 53(3):355-64. Available from: https://pesquisa.bvsalud.org/portal/resource/pt/lil-492538

4. Maeda MYS, Shirata NK, Longatto-Filho A, Cavaliere MJ, Santos $\mathrm{MM}$, Becker CC, et al. Influência da introdução da escova cervical para colheita de material cervicovaginal no programa de rastreamento de câncer ginecológico. RBM Ginecol Obstet. 1993; 4:55-8.

5. Stein MD, Fregnani JHTG, Scapulatempo C, Mafra A, Campacci N, Longatto-Filho A. Performance and reproducibility of gynecologic cytology interpretation using the FocalPoint system: results of the RODEO Study Team. Am J Clin Pathol. 2013 Oct; 140(4):567-71. Doi: 10.1309/AJCPWL36JXMRESFH 
6. Renshaw AA. Analysis of error in calculating the false-negative rate in the interpretation of cervicovaginal smears. Cancer. 1997 Oct; 81(5):264-71. Doi: $10.1002 /($ sici) 1097

0142(19971025)81:5<264::aid-cncr2>3.0.co;2-n

7. Ministério da Saúde (BR), Gabinete do Ministro. Portaria $n^{\circ} 3.388$ de 30 de dezembro de 2013. Redefine a Qualificação Nacional em Citopatologia na prevenção do câncer do colo do útero (QualiCito), no âmbito da Rede de Atenção à Saúde das Pessoas com Doenças Crônicas [Internet]. Brasília: Ministério da Saúde; 2013 [cited 2020 Aug 10]. Available from: http://bvsms.saude.gov.br/bvs/saudelegis

8. Ministério da Saúde (BR), Gabinete do Ministro. Portaria $n^{\circ} 613$ de 3 de março de 2017. Altera a Portaria no 3.388/GM/MS, de 30de dezembro de 2013, que trata da Qualificação Nacional em Citopatologia na prevenção do câncer do colo do útero (QualiCito) [Internet]. Brasília: Ministério da Saúde; 2017 [cited 2020 Aug 10]. Available from: https://www.in.gov.br/materia/

9. Branca M, Longatto-Filho, A. Recommendations on Quality Control and Quality Assurance in Cervical Cytology. Acta Cytologica. 2015; 59(5):361-369. Doi: 10.1159/000441515

10. Bortolon PC, Silva MAF, Corrêa FM, Dias MBK, Knupp VMAO, Assis M, et al. Quality Evaluation of Cervical Cytopathology Laboratories in Brazil. Rev Bras Cancerol [Internet]. 2012 [cited 2021 Jan 12]; 58(3):435-44. Available from: https://rbc.inca.gov.br/revista/index.php/revista/article/view/600

11. Contandriopoulos AP. Evaluating the institutionalization of evaluation. Ciênc Saúde Colet. 2006 July/Sep; 11(3):705-11. Doi: $10.1590 /$ S1413-81232006000300017

12. Furtado JP, Campos GWS, Oda WY, Onocko-Campos R. Health Planning and Evaluation: antagonism versus collaboration. Cad Saúde Pública. 2018 July; 34(7):e00087917. Doi: 10.1590/0102$311 \times 00087917$

13. Moraes PN, Iguti AM. Employee performance appraisal as a peculiar way of prescribing work: an analysis of PMAQ-AB. Saúde debate. 2013 July/Sep; 37(98):416-26. Doi: 10.1590/S010311042013000300005

14. Ministério da Saúde (BR), Instituto Nacional de Câncer José Alencar Gomes da Silva. Manual de gestão da qualidade para laboratório de citopatologia [Internet]. Rio de Janeiro: INCA; 2012 [cited 2020 Aug 10]. https://www.inca.gov.br/publicacoes/manuais/manual-de-gestaoda-qualidade-para-laboratorio-de-citopatologia

15. Ministério da Saúde (BR). Razão entre exames citopatológicos do colo do útero em mulheres de 25 a 59 anos e população feminina da mesma faixa etária e local de residência [Internet]. Brasília: Ministério da Saúde; 2018 [cited 2020 Aug 10]. Available from: http://dados.gov.br/dataset/wms_idsus_nt_rzcito

16. Ministério da Saúde (BR), Instituto Nacional de Câncer José Gomes da Silva. Nomenclatura Brasileira para Laudos Citopatológicos Cervicais [Internet]. Rio de Janeiro: INCA; 2012 [cited 2020 Aug 10]. Available from:

https://www.inca.gov.br/publicacoes/livros/nomenclatura-brasileirapara-laudos-citopatologicos-cervicais

17. Plewka J, Turkiewicz M, Duarte BF, Chaves MAF, Cestari C, Tartar DC. Evaluation of the quality indicators of cervical cytopathology laboratories. Rev Inst Adolfo Lutz. 2014; 73(2):140-7. Doi: 10.18241/0073-98552014731599

18. Ministério da Saúde (BR), Gabinete do Ministro. Portaria 1.338 de 08 de setembro de 2015. Habilita Laboratórios de Exames Citopatológicos do Colo do Útero [Internet]. Brasília: Ministério da Saúde; 2015 [cited 2020 Aug 10]. Available from: http://bvsms.saude.gov.br/bvs/saudelegis/gm/2015/prt1338_08_09 2015 .

19. Ministério do Planejamento, Orçamento e Gestão (BR), Instituto Brasileiro de Geografia e Estatística. Panorama do Brasil/Alagoas
[Internet]. Rio de Janeiro: IBGE; 2017 [cited 2020 Aug 10]. Available from: https://cidades.ibge.gov.br/brasil/al/panorama

20. Ministério da Saúde (BR), Instituto Nacional de Câncer José Alencar Gomes da Silva. Atlas On-line da Mortalidade. Rio de Janeiro: INCA; [cited 2021 Feb 17]. Available from: https://mortalidade.inca.gov.br/MortalidadeWeb

21. Ministério da Saúde(BR), Instituto Nacional de Câncer José Alencar Gomes da Silva. Plano de ação para redução da incidência e mortalidade por câncer do colo do útero: sumário executivo [Internet]. Rio de Janeiro: INCA; 2010 [cited 2020 Aug 10]. Available from: http://bvsms.saude.gov.br/bvs/

\section{Citação:}

Bandini HHM, Bandini CSM, Tavares RJD, Wyszomirska RMAF. Câncer de colo de útero: situação dos laboratórios em Alagoas. Rev. Port. Saúde e Sociedade. 2021;6(único):e02106004. Doi:10.28998/rpss.e02106004. 